\title{
Reviewing the Insights of Confinement and Social Distancing as Measures Involved in the Prevention of the COVID-19 Pandemic
}

\author{
Georges Pius Kamsu Moyo, Dany Hermann Ngwanou, \\ Daniel Armand Tague Kago, Nelly Kamgaing, \\ Ginette Claude Mireille Kalla, Andreas Chiabi, \\ Paul Olivier Koki Ndombo
}

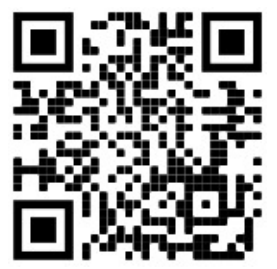

Faculty of Medicine and Biomedical Sciences, The University of Yaoundé I, Yaoundé, Cameroon

Corresponding Author: Georges Pius Kamsu Moyo

Email: kamsuzicfried@yahoo.fr Telp: (+237) 690206817

Article Info

Article history:

Received 20 August 2020

Received in revised form 31

August 2020

Accepted 1 September 2020

Keywords:

Confinement

Social Distancing

COVID-19

\begin{abstract}
Confinement and social distancing have been widely used in the prevention of the COVID-19 pandemic, as interventions consisting in reducing physical contact between individuals to prevent the spread of the disease. In order to demonstrate the pattern of these measures, we did a review of pertinent articles on the subject available online. We found that though confinement and social distancing significantly contributed to the mitigation of the COVID-19 infection in a number of countries worldwide, there however exist a dilemma in choosing between the expected benefits and adverse effects, especially when applied on a large scale. Thus considerations with regards to socioanthropological and politico-economic impacts should be considered in order to protect citizens, especially the vulnerable. Besides, population information, education and communication helps to increase adherence and observation of recommendations. However, further improvements need to be implemented in other to render these measures more bearable and less restrictive while ameliorating their efficacy.
\end{abstract}

\section{Introduction}

As of March 2020, the COVID-19 infection had claimed more than 20.000 lives in the world, with a case fatality rate close to 0.6-3.5\% (Kraemer et al., 2020; Wu \& McGoogan, 2020). After the intensification of the pandemic, the World Health Organization urged governments of all nations to consider the control of the disease within their boundaries as a top priority (WHO, 2020). As a matter of fact, public health authorities from all parts of the world had to make sure decision makers are aware, understand and consider the pandemic (Mark et al., 2020). Several optimal nation-wide medical, social, economic and political interventions have been undertaken to curb the COVID-19 pandemic, trying to eradicate the virus, while preserving human lives as much as possible (Kissler et al., 2020). Considerable advances in various research areas have been reported as well, in a myriad of aspects since the beginning of the crisis. Apart from the timing of intensive preventive measures reported in a number of studies conducted in China, the relative low prevalence of the epidemic in Africa prompted the development of further theories over the determinants of the disease's spread (Kissler et al., 2020). 
The influence of climatic factors and seasonal influences have been evoked, just as genetic and immunological make-up, probably associated with a lower average age of the population, reduced life expectancy and life-span (Kraemer et al., 2020; WHO, 2020; Kissler et al., 2020). On the other hand, some apostolic and spiritual-end hypotheses about the outbreak diverge from predictions on divine justice over mankind's evil acts calling for a change from perverted lifestyle, to apocalyptical predictions of the end of the world, calling for repentance (Curtis, 2020).

Whereas, from the scientific stand point, COVID-19 revealed once more the complex interconnectivity between the human organ systems as a whole and pathophysiological processes, as well as the flexibility of pharmacological concepts with major antimalarial drugs used for curing a viral infection, beside the necessity for palliative or supportive care in critically-ill patients (Zhang et al., 2020). Nevertheless, experience from the strongest leading nations such as the USA, China and Italy taught the world COVID-19 could lead to overwhelming of healthcare capacities of countries having highest resources, with infected people dying at home without being attended to (Moore \& June, 2020; Winfield, 2020; AP News, 2020). Indeed, in some cases, because of medical staff and equipment shortage, the increased demand for respiratory assistance, hospitals for patients' isolation, and protection material, exceeded capacities, causing healthcare burden due to difficulties to handle the excess demands (Kraemer, 2020; Moore \& June, 2020; Winfield, 2020; AP News, 2020). This in addition to the necessity to care for other patients having nothing to do with the epidemic, while protecting the staff and visitors from contamination (Heymann et al., 2013). Though drastic control measures substantially led to mitigation of the epidemic several months after in China, the said measures could not be integrally replicated in all nations worldwide due to varying parameters including Socio-anthropological and politico-economic factors mainly (Kraemer, et al., 2020; Kissler et al., 2020; Hawryluck, 2004). In a number of countries, these determinants were responsible for the deviation from ideal public health policies, causing on one hand differences as far the nature, the intensity and duration of preventive measures are concerned, and on the other hand subsequent variability in the dynamics of the disease transmission and spread [Kraemer et al, 2020; Kissler et al., 2020). Given that only 15\% patients manifest severe forms of the disease, while less than 5\% develop critical forms, it seemed more beneficial to admit such patients, meanwhile those with milder forms, not requiring critical care would be followed-up at home while being confined (Wu \& McGoogan, 2020; Mead, K., \& Johnson, 2004; Hauser et al., 2020; Verity 2020). Thus among various preventive measures in the prevention of COVID-19, confinement and social distancing though subjected to contextual modifications appear to be the most commonly used intervention to reduce the peak intensity of the pandemic thereby flattening the curve. These interventions permit to reduce the risk of overwhelming health systems and buys time for the development of efficient treatments or vaccines (Verity 2020). The rationale of this measure is founded on the fact that the virus to which Newton's laws of gravity apply as well because of its size, cannot remain suspended in the atmosphere and so relies on physical contact and human mobility for its dissemination and transmission (Kraemer, 2020). Therefore, restricting human mobility through confinement and social or physical distancing automatically breaks the chain of transmission. Nevertheless, confinement measures are best implemented when resources for populations' support and accompanying measures such as logistic, nutritional, financial, and psychological assistance are provided especially during large scale or community-wide confinement (Anderson et al., 2020).

\section{Background}


Social distancing in reality refers to physical distancing and may be better understood as such, in order to preserve social interactions. This is a group of actions including confinement measures (quarantine and isolation), mask wearing, forbidden handshaking and prohibited mass gathering, to prevent the transmission of a highly contagious disease, thereby reducing pressure on healthcare services [Anderson et al., 2020; ECDC, 2020). They are drastic measures which had no more been used to contain infectious diseases for more than fifty years before the recurrence of the Severe Acute Respiratory Syndrome (SARS) in 2003 (Risse, 1992; Twu et al., 2003; Toronto Public Health. 2003; Barbera, 2001).

Although they had periodically and successfully been used for centuries to control diseases such as cholera and plague, especially in travelers (Risse, 1992; Twu et al., 2003; Centers for Disease Control and Prevention (CDC), 2003; Mandavilli, 2003; Toronto Public Health, 2003; Barbera et al., 2001; Markel, 1995; Markel, 1993).

In effect, the mobility parameter "to have visited Wuhan in China" was seriously taken into account at the beginning of the COVID-19 epidemic for the "definition of cases". This was however gradually abandoned as the epidemic progressed to a global dimension, becoming a pandemic. But yet, motion restriction remained a pertinent aspect for the control of the epidemic, as human mobility seems to predict the magnitude of spread (Kraemer et al., 2020). Confinement may be adopted at various levels, varying from home quarantine indicated for contact persons assimilated to asymptomatic carriers of the infection, to hospital isolation for ill individuals. Whereas, the mobility restriction of citizens within cities and countries corresponds to community-wide confinement. Although there is no evidence that confinement and social distancing have led to the termination of an epidemic, it has however been shown that travel restriction, isolation and quarantine are particularly useful as confinement measures in the management of the early stage of an epidemic outbreak, especially when delimitated to the epicentre (Kraemer et al., 2020, Jones \& Carver, 2020).

While most high-income countries, opted for a total confinement approach with enough accompanying measures for citizens during the COVID-19, other governments mainly from middle and low-income countries opted for a "semi-confinement" approach. This consisted in appealing citizens to stay at home as much as possible, prohibiting mass gathering, crowding avoidance, and the closure of related environments including schools, bars, leisure and sports places (European Centre for Disease Prevention and Control, 2020). However, for all forms of confinement, barrier measures including face masks wearing, frequent handwashing, and social distancing including forbidden handshaking, one to two meters distancing between persons were strictly recommended (European Centre for Disease Prevention and Control, 2020). Nonetheless, Confinement and social distancing are however considered useless when the epidemic is widespread, given that community-borne immunity may soon be reached. Moreover, the dilemma of choosing between the expected benefits of confinement and its negative impact on freedom of movement, economy and socialization are as well important issues to deal with (Kraemer, 2020).

\section{Quarantine}

Quarantine may be defined as the restriction of persons exposed to a contagious disease but not manifesting signs nor symptoms (asymptomatic carriers), in order to prevent dissemination (Hawryluck et al., 2004; Anderson et al., 2020). It basically consists in separating individuals potentially exposed to an infection, and thus at risk for a disease, from the general community for the good of the majority (Hawryluck et al., 2004). Etymologically, quarantine originates from the 14th century during the prevention of plague, when infected-ships were forced to sit at anchor for a "quar-antine" to mean "forty-days" observation before disembarking 
(Hawryluck et al., 2004). Although its scientific basis has been reinforced with time, there however exist an associated historical stigma causing apprehensions due to the connotation of "sacrificing a few to save the majority" (Cetron \& Simone, 2004). The goal here is to suppress the exponential spread of the disease beyond care giving capacities, to a manageable proportion relatively easier to handle. The recommendation of quarantine restriction may be voluntary or mandatory according to the pathogen's infectivity, virulence and other environmental or human factors (Hawryluck et al., 2004; Anderson et al., 2020). Community-wide quarantine refers to the closing of community borders or the erection of real or virtual barriers also known as "cordon sanitaire" around a geographic area (Kraemer et al., 2020). Quarantine restriction is generally time-limited and short lasting according to factors of pathogenicity such as the pathogen's life cycle, its life span, mode of transmission, and the incubation period. The duration of quarantine was changed from 40 to 14 days, based on the incubation periods of diseases for which quarantine is recommended, as provided by the Public Health Service Act in the USA for instance (Anderson et al., 2020). Quarantine is one of the most feared and misunderstood, but effective method for controlling communicable disease outbreaks. It has been noticed that in a context of good information and communication over the necessity for people be on quarantine, more than $99.9 \%$ adhere to the recommendation (Anderson et al., 2020). In effect in a study conducted in the USA to assess the acceptation of quarantine, only 22 people out of 30000 expressed oppositions, requiring mandatory detainment (Kennedy \& Hamilton, 1997). Home quarantine is generally preferable and should be favoured as much as possible, just as voluntary quarantine should prevail over mandatory quarantine. Furthermore, the success of quarantine requires that individuals involved continue to be closely monitored with appropriate control measures within their place of quarantine, including regular temperature measurement at least twice daily (Anderson et al., 2020). It is recommended that health authorities should be notified at the earliest onset of signs and/or symptoms in quarantined persons, so as to enable their extraction and separation from others who are well (Hawryluck, 2004). Being on quarantine may create heavy psychological and financial repercussions in some persons, and so most public health policies advocate psychological support, food, water, household and medical supplies should be provided as accompanying measures (Anderson et al., 2020).

\section{Isolation}

Isolation may be described as the separation of ill persons affected with a particular disease, generally a contagious one, to either preserve non-affected individuals and/or favour the recovery of the patients. Contact isolation may as well be indicated for patients with multidrugresistant pathogens such as Methicillin-Resistant Staphylococcus Aureus (MRSA) (Abad et al., 2020). If not for the facts that isolation is meant for ill persons, and implemented with more rigor, with duration determined by the patient's recovery, it may be confused with quarantine and both terms falsely used interchangeably according to various contexts. A review of studies on the adverse effects of isolation in patients revealed a negative impact on mental wellbeing and behaviour, including higher scores for depression, anxiety, and anger (Stelfox et al., 2003; Hollenbeck et al., 1980). The reasons behind the negative effects of isolation probably stem from related feelings of uncertainty and loss of control reported in patients (Catalano et al., 2003; Gammon, 1998). A number of researchers suggest prior psychological preparation through education and information is necessary to decrease adverse effects in such patients as they better understand the essence of the process and cope with it (Holland et al., 1977; Knowles, 1993). During isolation, various dimensions of patients' care may be altered as well, mainly due to the fact that healthcare workers might spend less time in caring for isolated patients, this in line with formal or institutional recommendations, or because of an exaggerated fear of being contaminated. Furthermore, the use of gowns and special gloves could impede 
examination, manipulations, and be stigmatizing (Saint et al., 2003; Kirkland \& Weinstein, 1999). All these factors affect the level of patients' satisfactions, produces a negative impact on their safety, and cause up to eight-fold increase in adverse events pertaining to supportive care failure (Rees, 2000). However, there is evidence that patients' satisfaction is proportional to the quality of communication with the healthcare provider and so may be prevented (Rees et al 2000). The efficiency of contact isolation may be further reinforced with barrier measures.

\section{Objectives}

Social distancing measures including confinement as used in the prevention of the COVID-19 pandemic refers to efforts aiming at decreasing or interrupting the transmission of the infection in a population, through the minimization of physical contact between potentially infected individuals or population groups with high incidences and transmission rates, and those with no or low level of the disease transmission (European Centre for Disease Prevention and Control, 2020). From a public health perspective, they are intended to separate individuals exposed to a dangerous communicable disease from the general population and so may appear as a collective action for a common good (Anderson, 2020). It is intended to favour the recovery of already infected or exposed individuals and protecting others from inadvertent exposure (HHS, 2006). While "social distancing" involves various measures to reduce physical contact as a means to break the chain of transmission, it may reduce social contact and interpersonal interactions thereby affecting relationships. However, this is not the specific aim of the process. Social distancing measures could be reinforced or implemented concomitantly with other preventive measures such as contact tracing or tracking, and eventually testing and treating affected individuals. These interventions serve to protect vulnerable groups such as patients beyond 70 years, with comorbidities and at risk of severe outcomes, thereby decreasing the peak magnitude of the epidemic (European Centre for Disease Prevention and Control, 2020). More so, the prevention of the COVID-19 through social distancing has as advantage to have preserved health care capacities as well, preventing overwhelming of hospital's services.

\section{Initiating and stopping the measures}

In order to answer the question as to when confinement and other social distancing measures should be initiated, it may be necessary to recall that the success of such dispositions over a long period of time depends on the ability to make sure that people maintain some degree of social contact with relatives, though from a physical distance. For the past twenty years, internet-based communications, as well as Information and Communication technologies (ICTs) have proven to be essential for maintaining distant human relationships (European Centre for Disease Prevention and Control, 2020).

Community-wide confinement measures are best indicated when the transmission of an infectious disease in a given area is evident and the epidemiological links between new cases are unknown. Experience from observational and modelling studies from past epidemics such as the SARS pandemic in 2003, and evidences gathered from the progress of the COVID-19 with China as the epicentre reveal that the early, decisive, rapid, coordinated and comprehensive initiation of social distancing is probably more efficient in decelerating the spread of the virus than delayed interventions (Medicine Io, 2006; WHO, 2020). In fact, it is believed that the implementation of social distancing over one to three weeks at the beginning of the COVID-19 outbreak in China, may have yielded as much as 66 to $95 \%$ reduction of the spread of the disease, while reducing the number of affected areas as well (Lai et al., 2020). Therefore, the adoption of social distancing should be done as early as possible, when indicated. More so, before the finding of effective antivirals or preventive and therapeutic vaccines, confinement measures appeared to be the mainstays of containment strategies 
(Anderson, 2020). In as much as the layering of several measures likely increases the effectiveness of individual measures through additive or synergistic effects (Hatchett et al., 2007).

However, to render the course of social distancing more acceptable and bearable, the population should regularly be informed of various possible eventualities such as the extension of the measures if required by circumstances, the removal of some measures while adding some others as time goes on, and the possibility of re-imposing larger scale social distancing and restrictive measures in case there is resurgence of transmission following the lifting of measures (European Centre for Disease Prevention and Control, 2020). Today, various principles of public health strategies limit the use of quarantine and social distancing to extreme situations with highly contagious diseases (Anderson et al., 2020). When implementing such measures, it is important that an anticipated end date be communicated as soon as possible, based on scientific estimations or non-scientific but obvious realistic observation including political motivations (European Centre for Disease Prevention and Control, 2020). This enables psychological predisposition and mind setting based on targeting and planning, thereby improving resilience capacity and performance.

One of the limiting factors of social distancing measures seems to be the "time factor" which determines their success or failure. Besides, mass vaccination programs, the development of sufficient levels of immunity in the population through community exposition and contamination, also known as the "herd immunity" appears to be the end point of these measures, when they could become useless and may be stopped (European Centre for Disease Prevention and Control, 2020).

\section{Considerations}

Some considerations should be taken into account when adopting social distancing measures, given that the natural pattern of the epidemic, the socioeconomic, political and legal contexts may vary from one nation to another. As such the balance between scientific justification, social implications of such measures including norms and values associated with the freedom of movement should be compared with the public reactions with respect to restrictive constrains and exposition risk (Mori, 2020). Other factors to be considered may also include feasibility of measures, the time factor, institutional parameters and international pressures.

On the other hand, the legal and ethical considerations are well established as far as social distancing as a preventive measure during epidemic outbreaks is concerned. In most countries worldwide, it is the responsibility of public health authorities in conformity with national and international legal principles to prevent the introduction, the transmission and the spread of communicable diseases from foreign countries or from one geographical area to another within a country (Anderson, 2020; Council on Ethical and Judicial Affairs, 2005). They determine the conditions and principles required for imposing confinement measures including quarantine, isolation and other social distancing measures with respect to the hierarchy of juridical norms and considerations. In most countries, individuals may however have to some extends, the right to appeal within ten days for legal procedures and hearing before competent instances for the release from quarantine, and relief from violations due its conditions (Anderson et al, 2020; United Nations, Economic and Social Council, 1985; WHO, 2005). The bioethical conflict which may arise between policies intended to protect the community and the necessity to respect principles regarding the care of individual patients, may be resolved by ensuring communities' information. This in order to enable their participation in the development of policies, programs, priorities, their accessibility to basic resources, and conditions necessary for health and protection of confidentiality (Anderson et al, 2020). 
In the same vein, ethical recommendations require that when implementing extreme measures, decisions should be rooted in scientific rationale, proven effectiveness, efficacy of the said measure, and the public necessity. Likewise, proportionality in minimal infringement, justice, fairness and reciprocity should be assured for all (Mori, 2020). In such a scheme, the implementation of various measures would be applicable to all and not differentiating between individuals' social or economic classes.

Furthermore, a comprehensive communication on "risks" should be developed in order to present the rationale justifying the implementation of various measures. This should aim to encourage people to take actions, at least at the individual level for self-protection (European Centre for Disease Prevention and Control, 2020). A communication targeting all social strata, including minorities may further enhance adhesion and large scale implication. All these factors need to be considered and anticipated as well as mitigation planning.

Restrictive measures due to confinement and social distancing lead to the limitation of human mobility and eventually short or medium-term financial burden (Cetron \& Landwirth, 2005). Therefore, financial compensation and support for income and employment loss would help to reinforce adherence to public health measures (European Centre for Disease Prevention and Control, 2019). However, some degree of business continuity as far as critical and essential services is concerned, is recommended. This is the case of healthcare services, pharmacies, fire, water, gas and electricity sectors for example, for which interruption may cause high societal consequences (Willem et al., 2020; International Organization for Standardization, 2019).

Enough support for people and communities subjected to social distancing measures should be provided to ease adherence. Such support consists in ensuring the provision of necessary services and supplies such as food, medication, and healthcare especially for vulnerable subpopulations (DiGiovanni et al., 2004; Barbera et al., 2001). As for smaller children, including contact or infected neonates and toddlers, they could be accompanied by a parent or a care giver during confinement, with the reinforcement of barrier measures to prevent contamination. Contact with relatives through internet-based systems should be favoured in order to preserve some level of socialization. Home practice of physical activity and good feeding should be promoted as well (UNESCO, 2020).

More so, confinement measures may further be strengthened by the promotion of solidarity and mutual community support. This has been the case in some countries where people from their homes were found singing together, comforting each other, applauding and encouraging health workers from their apartment buildings, through windows or balconies, hanging banners carrying encouraging messages. Furthermore, various industries and commercial enterprises could assist the vulnerable with food, face masks and other donations, just to name a few (European Centre for Disease Prevention and Control, 2020).

Stigmatization should be prevented as much as possible, given that evidence from previous epidemics management reveal people under quarantine and isolation may suffer stigmatization even when proven not to be affected by the disease (Desclaux et al., 2017). Such experiences may reduce adherence and cause long-term psychosocial frustrations (Brooks et al., 2020). It is therefore necessary for health authorities to proactively address this issue through the promotion of solidarity, by putting forward the fact that everyone is potentially at risk of being infected and that only common efforts would help protect everyone (Brown et al., 2003).

Control processes and regular assessments with regards to the impact of various measures implemented, their efficacy as well as the population's adherence should be constantly carried out to enable consequent adjustments (Willem et al., 2020; ECDC, 2020). 


\section{Benefits and advantages}

Confinement and social distancing during the COVID-19 pandemic significantly contributed to curb the disease's spread in most countries, if not all. Mankind adaptability and flexibility was proofed, but succeeded through readjustment of habits, changing usual modes of functioning and established protocols, directing attention towards other interests and possibilities. Confinement and other social distancing measures have however been implemented with varying rigor and extends from one state to another, with relative effectiveness and efficacy (Willem et al., 2020; European Centre for Disease Prevention and Control, 2020). While it was total and community-wide in a number of developed countries, it was rather milder and less restrictive in most developing countries, especially in Africa. This permitted to prevent cataclysmic predictions while maintaining some degree of socioeconomic preservation. However, the majority of high-income countries could cope with country-wide confinement without unbearable adverse effect, and so were more prone to large scale confinement over three months and more, promoting populations' mutual support and solidarity (European Centre for Disease Prevention and Control, 2020). On the other hand, the restriction of international transaction and exchanges allowed for resilience, self-reliance, emulation and development especially in low-income countries. As such, considerable ameliorations and progress in scientific research, improvement of health systems and facilities occurred. The COVID-19 epidemic outbreak has equally strengthened some aspects of international relations, bringing nations together, in the sense of putting in efforts in a bid to find common solutions through collaboration, and contextual-motivated solidarity (Anderson et al., 2020; European Centre for Disease Prevention and Control, 2020; Willem et al., 2020; ECDC, 2020).

During these periods of restricted human mobility and decreased outhouse job occupation, most family links and relationships were reinforced, as parents became more available and hence closer to their wives and children (Cetron \& Landwirth, 2005; Mori, 2020; Council on Ethical and Judicial Affairs, 2005). Likewise working at home helped to relieve stress in some persons, with necessary and sufficient rest, producing favourable effects on mental health status. Moreover, increased online services improved the knowledge and use of digital Information and Communication Technologies (ICTs) (European Centre for Disease Prevention and Control, 2020). More so, confinement provided enough time for other interests including introspective assessment, contributing to personal development based on spirituality and religion, necessary to build up faith, with prayer as an appeal for divine salvation. Forbidden handshaking and regular hand washing helped to increase good hygiene practices. Furthermore, the dedication of television programming and media advertisement, as well as the free access to written articles about COVID-19 also contributed for public education on the topic (Anderson., 2020).

\section{Burden and inconveniences}

Confinement and social distancing has led to considerable degradation of socialization and social interactions, promoting individualism, and egoistic self-reliance and self-sufficiency (Anderson., 2020, European Centre for Disease Prevention and Control, 2020). The significant degradation of the economic tissue comprising financial limitations, large scale unemployment, increased rate of job loss, reduced circulation of commodities, and the limitation of services drastically impacted incomes and revenues (Anderson., 2020, European Centre for Disease Prevention and Control, 2020; Council on Ethical and Judicial Affairs, 2005). Eventually, the political situation of some unstable nations got worsened, due to the increase of contestations with regards to administration strategies, decision making, and failure of governmental systems. More so, international cooperation and exchanges have been reduced as well, because 
of confinement measures (Anderson et al., 2020; European Centre for Disease Prevention and Control, 2020).

Other undesirable repercussions of confinement measures involved national and international missed appointments, academic and professional cancellation or cessation of activities and services. Moreover, even though confinement and social distancing have been effective in reducing the spread of infectious diseases, it is however believed a 100\% implementation may never be reached, and so the possibility of repeating the process due recontamination exists. More so, prolonged confinement may lead to missed family planning, increased birth rates and eventually demographic expansion. Some mathematical projections of modelling research predict the pandemic may take up to five years before being eradicated from the face of the earth, when considering social distancing alone (Kraemer et al., 2020). In this case, it would be quite a long-lasting process, difficult to bear.

Furthermore, confinement and quarantine measures in some cases may lead to the violation of individual rights such as poorly justified deprivation of freedom and access to basic necessities including health assistance. Moreover, the difficulty to follow-up patients with known preexisting comorbidities including cardiovascular and psychological backgrounds, may be responsible for the onset of decompensation and degradation of previously stabilized health conditions (European Centre for Disease Prevention and Control, 2020). These decompensation phenomena could be aggravated by confinement-related stress and anxiety, the restriction of needs and rights, as well as the increased rates of obesity due to reduced human mobility and physical exercises (Anderson et al., 2020). On the other hand, there may be difficulties with the observation of various restrictions in some vulnerable subjects such as rebel adolescents, the sedentary old, the mentally unstable and the handicapped (Kraemer, 2020; Anderson et al., 2020, European Centre for Disease Prevention and Control, 2020). Moreover, by the end of the confinement process, citizens may drastically neglect further recommendations or restrictions with carelessness, and excessive loosing up due to a period of long lasting privation. Such an "obstacle removal syndrome" would therefore be in line with or occur as a result of the "forbidden fruit theory". In such a context, this would be a risk factor for recontamination and restarting the process all-over.

\section{Perspectives}

In periods of serious lives claiming by epidemics, confinement measures including quarantine and isolation should be mandatory for symptomatic individuals and contact subjects, with written order and recommendation carrying the highest authority's signature, so as to improve the control of cases and preserve the lives of many (Anderson et al., 2020; European Centre for Disease Prevention and Control, 2020). Resources should be made available to support the vulnerable including the sick, the old, the poor, and the disable. Moreover, barrier measures such as one to two meters physical distancing, face masks wearing, regular hand washing, and forbidden hand shaking among individuals should be associated and penalty constraints established to achieve rigorous observation (Kissler et al., 2020; Anderson et al., 2020; European Centre for Disease Prevention and Control, 2020).

Confinement places including hospitals, workplaces and houses should regularly be disinfected to reduce viral concentration in the area and hence infectivity. A network of psychological support should be put in place to care for the confined. More so, the reinforcement of targeted information, communication and debates relative to daily statistical updates, disease transmission and preventive measures, may serves a great purpose in such contexts (Anderson et al., 2020; Markel, 1993; Knowles, 1993; Rees et al., 2000). 
Education should be continued by conceiving detailed and explicit electronic lessons and teachings, as well as video conferences especially for secondary, high school and university students. These could be made available and accessible to students through the internet, such that they can be downloaded and read at any moment. In the same vein, teleworking tasks could be developed and made feasible for workers on confinement, so as to reduce the rate of unemployment, job loss and financial limitations (Kissler et al., 2020; Anderson et al., 2020; European Centre for Disease Prevention and Control, 2020). Some degree of physical distancing may still be observed on workplaces while delivering the minimal necessary services, especially when non-essential staff are oriented towards teleworking and homeworking (European Centre for Disease Prevention and Control, 2020). In a similar manner, efforts should be made in order to preserve permanent health services to attend people with diseases different from the ongoing epidemic (Kissler et al., 2020; Anderson et al., 2020; European Centre for Disease Prevention and Control, 2020). Furthermore, patients should be encouraged to contact their physician as much as possible before each visits, so that the necessity of a visit could be appreciated and the required predispositions taken.

Social interactions could be virtualized during this period in order to maintain a certain level of socialization (European Centre for Disease Prevention and Control, 2020). For such makeups, the closer to reality they seem, the better the impact produced. They could include large screen viewing of family reunions, meetings, parties, and concerts, sufficiently charged with emotions to generate feelings of attachment. Due to forbidden mass gathering, matches and sports competitions should be diffusely broadcasted in real time, including through television, radio stations and the internet (Anderson., 2020; European Centre for Disease Prevention and Control, 2020). Pre-recorded supporters' noises may help to motivate competitors, players, and tele viewers, by producing sensational effects. Likewise, religious activities may be followed and performed at home as well, to avoid crowding.

\section{Conclusion}

It appears that confinement and social distancing are effective and efficient in reducing epidemic dissemination, and should be implemented as early as possible during deadly outbreaks. Their effectiveness may be time-limited, depending on the development of natural community-borne immunity or a vaccine. The negative impacts of these measures are mainly the restriction of human mobility, as well as human interactions and this may affect almost all aspects of human activities, impacting the lives of nations as a whole. However, as a response, preserving some level of socioeconomic functioning, while strengthening the necessary support to citizens through the implementation of accurate accompanying measures could help to prevent further misery and the escalation of health issues.

\section{Acknowledgements}

All collaborators to this project.

\section{Funding}

Private

\section{Conflict of Interest}

The authors declare that they have no competing interest.

\section{References}

Abad, C., Fearday, A., \& Safdar, N. (2010). Adverse effects of isolation in hospitalised patients: a systematic review. Journal of hospital infection, 76(2), 97-102. 
Anderson, R. M., Heesterbeek, H., Klinkenberg, D., \& Hollingsworth, T. D. (2020). How will country-based mitigation measures influence the course of the COVID-19 epidemic?. The Lancet, 395(10228), 931-934.

AP News. (2020). COVID-19 infections rise in New York with peak weeks away. AP NEWS, 25 March 2020; Available from: https://apnews.com/7c7563cb82626a4042797c6aa6da260a.

Barbera, J., Macintyre, A., Gostin, L., Inglesby, T., O'Toole, T., DeAtley, C., \& Layton, M. (2001). Large-scale quarantine following biological terrorism in the United States: scientific examination, logistic and legal limits, and possible consequences. Jama, 286(21), 2711-2717.

Barbera, J., Macintyre, A., Gostin, L., Inglesby, T., O'Toole, T., DeAtley, C., \& Layton, M. (2001). Large-scale quarantine following biological terrorism in the United States: scientific examination, logistic and legal limits, and possible consequences. Jama, 286(21), 2711-2717.

Brooks, S. K., Webster, R. K., Smith, L. E., Woodland, L., Wessely, S., Greenberg, N., \& Rubin, G. J. (2020). The psychological impact of quarantine and how to reduce it: rapid review of the evidence. The Lancet.

Brown, L., Macintyre, K., \& Trujillo, L. (2003). Interventions to reduce HIV/AIDS stigma: what have we learned?. AIDS education and prevention, 15(1), 49-69.

Catalano, G., Houston, S. H., Catalano, M. C., Butera, A. S., Jennings, S. M., Hakala, S. M., \& Laliotis, G. J. (2003). Anxiety and depression in hospitalized patients in resistant organism isolation. Southern medical journal, 96(2), 141-146.

Centers for Disease Control and Prevention (CDC). (2003). Use of quarantine to prevent transmission of severe acute respiratory syndrome--Taiwan, 2003. MMWR. Morbidity and mortality weekly report, 52(29), 680.

Cetron, M., \& Landwirth, J. (2005). Public health and ethical considerations in planning for quarantine. The Yale journal of biology and medicine, 78(5), 329.

Cetron, M., \& Simone, P. (2004). Battling 21st-century scourges with a 14th-century toolbox. Emerging infectious diseases, 10(11), 2053.

Council on Ethical and Judicial Affairs. (2005). Report of the CEJA: The Use of Quarantine and Isolation in Public Health Interventions. January, 2005. Report of the Council on Ethical and Judicial Affairs. CEJA Report 1-1-05. Available from: http://www.amaassn.org/ama1/pub/upload/mm/369/ceja_recs_1i05.pdf

Curtis, W. (2020). Hart. Spiritual Lessons from the Coronavirus Pandemic. Journal Religi Health, 1(2).

Desclaux, A., Badji, D., Ndione, A. G., \& Sow, K. (2017). Accepted monitoring or endured quarantine? Ebola contacts' perceptions in Senegal. Social science \& medicine, 178, $38-45$.

DiGiovanni, C., Conley, J., Chiu, D., \& Zaborski, J. (2004). Factors influencing compliance with quarantine in Toronto during the 2003 SARS outbreak. Biosecurity and bioterrorism: biodefense strategy, practice, and science, 2(4), 265-272.

ECDC. (2020). European Centre for Disease Prevention and Control. Guidelines for the use of non-pharmaceutical measures to delay and mitigate the impact of 2019-nCoV. ECDC: Stockholm. 
ECDC. (2020). European Centre for Disease Prevention and Control. Guidance on community engagement for public health events caused by communicable disease threats in the EU/EEA. Stockholm: ECDC.

European Centre for Disease Prevention and Control. (2019). Community and institutional public health emergency preparedness synergies - enablers and barriers. Case studies on acute gastroenteritis in two EU/EEA Member States. Stockholm: ECDC.

European Centre for Disease Prevention and Control. (2020). Considerations relating to social distancing measures in response to COVID-19 - second update. Stockholm: ECDC.

Gammon, J. (1998). Analysis of the stressful effects of hospitalisation and source isolation on coping and psychological constructs. International journal of nursing practice, 4(2), 84-96.

Hatchett, R. J., Mecher, C. E., \& Lipsitch, M. (2007). Public health interventions and epidemic intensity during the 1918 influenza pandemic. Proceedings of the National Academy of Sciences, 104(18), 7582-7587.

Hauser, A., Counotte, M. J., Margossian, C. C., Konstantinoudis, G., Low, N., Althaus, C. L., \& Riou, J. (2020). Estimation of SARS-CoV-2 mortality during the early stages of an epidemic: a modelling study in Hubei, China and northern Italy. medRxiv.

Hawryluck, L., Gold, W. L., Robinson, S., Pogorski, S., Galea, S., \& Styra, R. (2004). SARS control and psychological effects of quarantine, Toronto, Canada. Emerging infectious diseases, 10(7), 1206.

Heymann, D. L., Mackenzie, J. S., \& Peiris, M. (2013). SARS legacy: outbreak reporting is expected and respected. The Lancet, 381(9869), 779-781.

HHS. (2006). Pandemic Influenza Plan, Appendix Principles of Modern Quarantine. Washington: US Department of Health and Human Services; 2006. Available from: http://www.hhs.gov/pandemicflu/plan/

Holland, J., Plumb, M., Yates, J., Harris, S., Tuttolomondo, A., Holmes, J., \& Holland, J. F. (1977). Psychological response of patients with acute leukemia to germ-free environments. Cancer, 40(2), 871-879.

Hollenbeck, A. R., Susman, E. J., Nannis, E. D., Strope, B. E., Hersh, S. P., Levine, A. S., \& Pizzo, P. A. (1980). Children with serious illness: Behavioral correlates of separation and isolation. Child Psychiatry and Human Development, 11(1), 3-11.

International Organization for Standardization. (2019). ISO 22301:2019 Security and resilience - Business continuity management systems - Requirements. Geneva: ISO; 2019.

Jones, N., \& Carver, C. (2020). Are interventions such as social distancing effective at reducing the risk of asymptomatic healthcare workers transmitting COVID-19 infection to other household members. United Kingdom: Centre for Evidence Based Medicine.

Kennedy, P., \& Hamilton, L. R. (1997). Psychological impact of the management of methicillin-resistant Staphylococcus aureus (MRSA) in patients with spinal cord injury. Spinal Cord, 35(9), 617-619.

Kirkland, K. B., \& Weinstein, J. M. (1999). Adverse effects of contact isolation. The Lancet, 354(9185), 1177-1178. 
Kissler, S. M., Tedijanto, C., Goldstein, E., Grad, Y. H., \& Lipsitch, M. (2020). Projecting the transmission dynamics of SARS-CoV-2 through the postpandemic period. Science, 368(6493), 860-868.

Knowles, H. E. (1993). The experience of infectious patients in isolation. Nursing Times, 89(30), 53-56.

Kraemer, M. U., Yang, C. H., Gutierrez, B., Wu, C. H., Klein, B., Pigott, D. M., \& Brownstein, J. S. (2020). The effect of human mobility and control measures on the COVID-19 epidemic in China. Science, 368(6490), 493-497.

Lai, S., Ruktanonchai, N. W., Zhou, L., Prosper, O., Luo, W., Floyd, J. R., \& Yu, H. (2020). Effect of non-pharmaceutical interventions for containing the COVID-19 outbreak in China. medRxiv.

Mandavilli, A. (2003). SARS epidemic unmasks age-old quarantine conundrum, 9:487.

Mark, K., Steel, K., Stevenson, J., Evans, C., McCormick, D., Willocks, L., ... \& Koch, O. (2020). Coronavirus disease (COVID-19) Community Testing Team in Scotland: A 14-day review, 6 to 20 February 2020. Eurosurveillance, 25(12), 2000217.

Markel, H. (1993). Cholera, quarantines, and immigration restriction: the view from Johns Hopkins, 1892. Bulletin of the History of Medicine, 67(4), 691-695.

Markel, H. (1995). Knocking out the Cholera: Cholera, Class, and Quarantines in New York City, 1892. Bulletin of the History of Medicine, 69(3), 420-457.

Mead, K., \& Johnson, D. L. (2004). An evaluation of portable high-efficiency particulate air filtration for expedient patient isolation in epidemic and emergency response. Annals of emergency medicine, 44(6), 635-645.

Medicine Io. (2006). Modeling community containment for pandemic influenza: a letter report. Washington, DC: Press: National Academies

Moore, J. B., \& June, C. H. (2020). Cytokine release syndrome in severe COVID19. Science, 368(6490), 473-474.

Mori, I. (2020). Poll of 8 countries finds majority say coronavirus poses a threat to the world 2020. Available from: https://www.ipsos.com/ipsos-mori/en-uk/poll-8-countriesfinds-majority-say-coronavirus-poses-threat-world.

Rees, J., Davies, H. R., Birchall, C., \& Price, J. (2000). Psychological effects of source isolation nursing (2): patient satisfaction. Nursing Standard (through 2013), 14(29), 32.

Risse, G. B. (1992). A Long Pull, A Strong Pull, And All Together: San Francisco And Bubonic Plague, 1907-1908. Bulletin of the History of Medicine, 66(2), 260-286.

Saint, S., Higgins, L. A., Nallamothu, B. K., \& Chenoweth, C. (2003). Do physicians examine patients in contact isolation less frequently? A brief report. American journal of infection control, 31(6), 354-356.

Stelfox, H. T., Bates, D. W., \& Redelmeier, D. A. (2003). Safety of patients isolated for infection control. Jama, 290(14), 1899-1905.

Toronto Public Health. (2003). Severe acute respiratory syndrome (SARS), 2003 May 29. Available from: http://www.toronto.ca/ health. 
Twu, S. J., Chen, T. J., Chen, C. J., Olsen, S. J., Lee, L. T., Fisk, T., \& Wu, Y. C. (2003). Control measures for severe acute respiratory syndrome (SARS) in Taiwan. Emerging infectious diseases, 9(6), 718.

UNESCO. (2020). Covid-19 and Education 2020. Available from: https://en.unesco.org/themes/education-emergencies/coronavirus-school-closures.

United Nations, Economic and Social Council (UNESCO). (1985). Siracusa principles on the limitation and derogation provisions in the international covenant on civil and political rights, U.N. Doc. E/CN.4/1985/4, Annex (1985). United Nations. Available from: http://hrlibrary.umn.edu/instree/siracusaprinciples.html.

Verity, R., Okell, L. C., Dorigatti, I., Winskill, P., Whittaker, C., Imai, N., ... \& Dighe, A. (2020). Estimates of the severity of coronavirus disease 2019: a model-based analysis. The Lancet infectious diseases.

WHO. (2005). International Health Regulations. 3 ed. Geneva: World Health Organization.

WHO. (2020). COVID-19: Top priority must be on containment, insists WHO's Tedros: UN News, 2020. Available from: https://news.un.org/en/story/2020/03/1058461.

WHO. (2020). Report of the WHO-China Joint Mission on Coronavirus Disease 2019 (COVID-19). Geneva: World Health Organization.

Willem, L., Van Hoang, T., Funk, S., Coletti, P., Beutels, P., \& Hens, N. (2020). Socrates: An online tool leveraging a social contact data sharing initiative to assess mitigation strategies for COVID-19. medRxiv.

Winfield, N. (2020). Not a wave, a tsunami. Italy hospitals at virus limit, AP NEWS, 13 March 2020. Available from: https://apnews.com/a4497f31bf5dbc1ff263e4263fc9f69e.

Wu, Z., \& McGoogan, J. M. (2020). Characteristics of and important lessons from the coronavirus disease 2019 (COVID-19) outbreak in China: summary of a report of 72 314 cases from the Chinese Center for Disease Control and Prevention. Jama, 323(13), 1239-1242.

Zhang, C., Wu, Z., Li, J. W., Zhao, H., \& Wang, G. Q. (2020). The cytokine release syndrome (CRS) of severe COVID-19 and Interleukin-6 receptor (IL-6R) antagonist Tocilizumab may be the key to reduce the mortality. International journal of antimicrobial agents, 105954. 